\title{
An Advance loT based Road Traffic Manipulation System
}

\author{
A. S. Zaforullah Momtaz \\ Assistant Professor \\ Dept of CSE \\ University of Asia Pacific
}

\author{
Rakib UI Haque \\ Undergraduate Student \\ Dept of CSE \\ University of Asia Pacific
}

\author{
Nusrat Jahan \\ Undergraduate Student \\ Dept of CSE \\ University of Asia Pacific
}

\begin{abstract}
WSN Traffic Control appliance is a well-turned-out solution of controlling the most disaster state of affairs occurs in highway, streets and many more. It is capable of manipulating vehicle or traffic so that it may diminish times in road rather than sitting inactive for extensive traffic troubles known as traffic jam. The proposed project introduces an IoT based traffic manipulation system and fleeting data through cloud in order to observe and maintain traffic at any time from any distinct places. It contains traffic lamps which passes major data through wireless sensor network to database of the workstation. The server calculates the percentage of compactness of traffic and then the data is provided both to the traffic signals and users via IoT shields as well as smart phones respectively. It also generates automated time schedule which manipulates traffic signals so that it can provide the proper outcome for the passing vehicles.
\end{abstract}

\section{General Terms}

IoT, Traffic Control System, Android, iOS, Mobile App, WSN, Artificial Intelligence.

\section{Keywords}

Cloud Server, Arduino Nano, ESP-8266 and HC-06 Shield.

\section{INTRODUCTION}

The Internet of things [1] (IoT) is the communication media of a large number of physical devices, vehicles, home appliances, and other items embedded with electronics, software, sensors, actuators, and connectivity. An IoT device is capable to connect, collect and communicate data, creating opportunities for more direct integration of the physical world on basis of computing systems which results in efficiency improvements, economic benefits, and abridged human exertions.

The uses of internet linked devices are increasing very rapidly. The ability to network embedded devices with fewer amounts of memory, CPU and power resources means that IoT finds applications in nearly every field. IoT's most impressive landmark in current time is the rapid development of devices linked and maintained with the help of www or internet [2]. This is one of the reasons; our research focuses on the IoT based traffic manipulation system. This paper is all about the way we can control the traffic system with IoT.

IoT-centric traffic manipulation system could be the best way to optimize the traffic jam because all the information like the number of vehicles in each road and there passing time will be presented in work station [3], then find out the best solution on a regular routine work. Also, the job will be done by the CCTV [4] if required only to manage traffic flows and providing advice regarding traffic congestion.

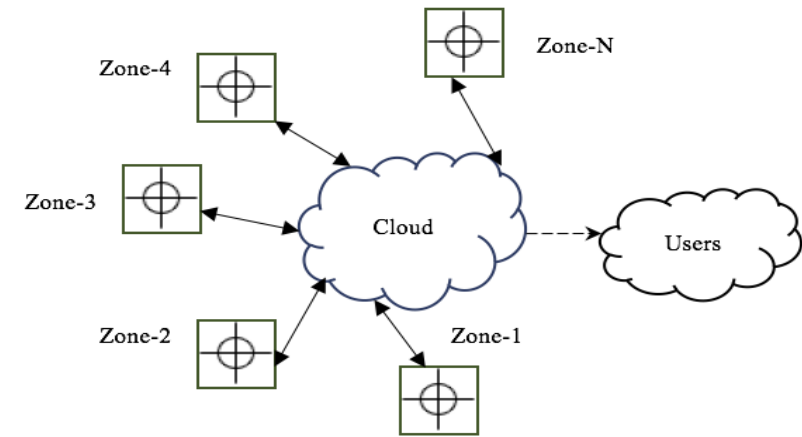

Fig 1: IoT-centric traffic manipulation system.

In the proposed system, 3 different modules have been considered like mobile applications to send and get traffic data update, a server to manipulate the data as well as the embedded lamp posts to reflect the status. The server collects information from traffic policemen and then forward the produced responses to all the users as well as the embedded [5] lamp posts to multiple nodes of the city.

\section{LITERATURE REVIEW}

The number of vehicles is rapidly increasing day by day. Thus, the manipulation of transports needs an on-demand upgradation to minimize the required time and fuel consumption, as well as maximize the engine lifetime while passing miles of pathways every day. In these circumstances, many researchers have been working on road traffic manipulation system. There are several techniques to manipulate road traffic.

To control the road traffic, "IoT based Traffic Light Control System using Raspberry Pi" [6] - authors have proposed an IoT based system which can make decisions for luminous [7] control depending on the intensity of light. Both of the 'day' and 'night' mode can be identified by setting up a fixed intensity value on LDR [8] sensor and lights of traffic lamp post can be controlled by the IR [9] sensor. Solar cells [10] are used as a power source and there is a backup DC current supply for the emergency situation. The total system can work without traffic police and traffic signals can be controlled automatically and monitor the entire system through the internet by installing CC camera on basis of a fixed time to with equal priority to all the lanes.

In another paper "IoT based street lighting and traffic management system" [11] - authors have discussed that current traffic control systems are dependent on fixed signal timing. They proposed an autonomous traffic compactness in which signal timings are updated depending on the number of vehicles in each lens. WI-FI transceiver shield [12] is used to 
transmit the number of traffic of the current system to the next traffic signals. Signals are updated depending on the traffic density of the past signals. Also, they introduced an image processing technique to count the number of vehicles which is the main technique of this paper to hold or release a lane.

Both of the proposed systems have focused on the timing and the number of vehicles on the roads, but a more generalized solution is required for a suddenly crowded traffic situation and for special traffic situations including emergency ambulance, firefighters or VIP Passing etc. Thus, the proposed system is capable to handle this task both in the manual by the traffic policemen and automated process via the traffic manipulation cloud workstation using IoT.

\section{FUNCTIONAL ACTIVITIES}

The proposed IoT based road traffic manipulation system interconnects the traffic police, pedestrians as well as all the vehicles passing by the roads in terms of maintaining the traffic lamp posts. This system is maintained and managed by the self-driven cloud server. Traffic polices are the key person who inserts the input manually by their vision as well as by the guidance of the workstation. Pedestrians and passing vehicles get only road traffic update in their personal smart phones and/or wrist watches.

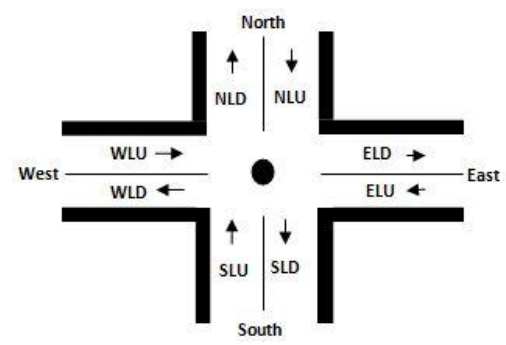

Fig 2: 4-way traffic control system.

In this paper, 4-way traffic control system is considered as an example to explain the operations of modules of this ecosystem. For Thus, the entire system can be classified in some small sections described as below:

\subsection{Traffic Signal Generation}

Generation of Traffic signal can be done by two different ways in terms of both in the manual process by the assigned traffic police man as well as the automated process generated by the cloud server. In the proposed system, traffic signal generation can be done by mobile apps. Update from the cloud server only shows as suggestion to the traffic policeman who acts as the data input end user and decision maker of the current signal status. The real traffic signal lamp post reflects the update, according to the decision of this user end.

\subsection{Signal Status Update}

The cloud server acts as central traffic manipulation system which connects all the traffic police apps, traffic signal lamp posts as well as the traffic mapping update apps. This unit takes the number of vehicles passing by the roads along their weights, speeds and directions as input. It also takes the traffic signal input generated by the traffic police men instantly. The main task of the server is to analyze the input data and generate the best suitable update result for a smooth travel. The generated result reflects the status update to the end users according to their accessibility.

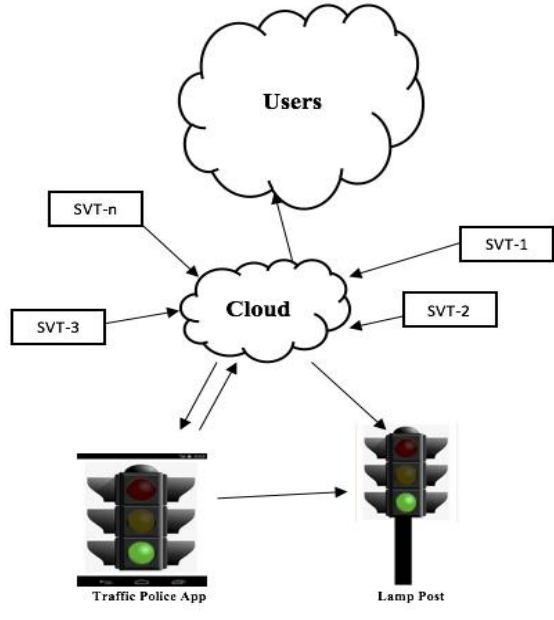

Fig 3: Data sharing of mobile apps, cloud server and embedded traffic lamp posts.

\subsection{Real Time Traffic Status}

All the end user of road traffic ecosystem requires the traffic mapping update at real time. This module is a general mobile app which is accessible by all the users passing by the road including passing vehicles, pedestrians, and traffic police men at all the crossing circles. This application provides the necessary update about the present red signals, upcoming red signals as well as approximate waiting time from the current location to the destination.

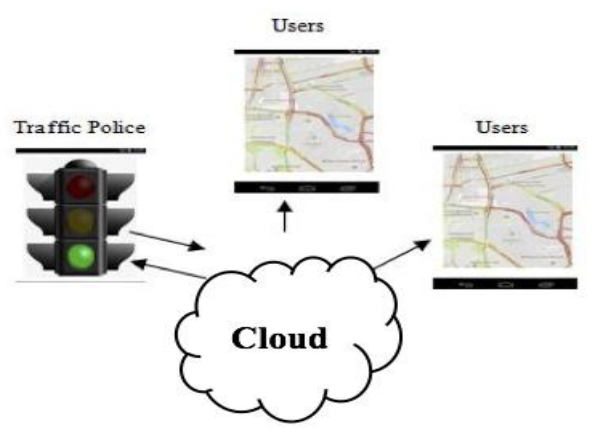

Fig 4: Cloud generates traffic mapping update after receiving signal from different location.

\section{TECHNICAL OVERVIEW}

The proposed system requires three different platforms to work with including mobile OS like Android [13] and iOS [14], Apache Server [15] as well as the embedded lamp post to present the traffic signals. There are two different types of mobile apps has been introduced to perform data input operations by the traffic police men as well as getting the traffic status update obtained from the cloud server which connects both of the apps mentioned above including the embedded lamp post. The entire ecosystem performs several technical operations to exchanges information from one point to another.

\subsection{Traffic Signal Post:}

The traffic lamp post prototype is designed using an Arduino Nano [16] board which uses two different communication terminals like Bluetooth Shield for the local network and WiFi Shield for cloud network. Also, Serial LED Set is used to demonstrate the traffic light. The purpose of Bluetooth module is to connect the lamp post to the traffic police app to 
get the signal command. And the Wi-Fi Shield is required to communicate with cloud workstation. To make the prototype simple, Arduino Nano board is used to demonstrate 4 Lamp Posts, HC-05 [17] Bluetooth Shield and ESP-8266 [18] for handling the cloud network.

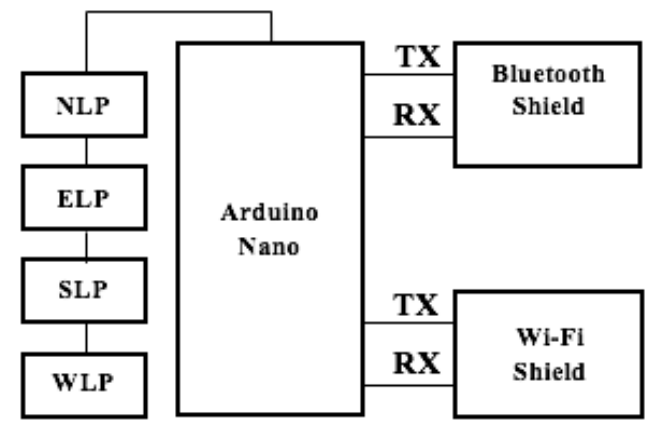

Fig 5: Controlling lamp posts (LP) of 4 lanes with Arduino Nano board.

As the traffic policeman app is in the Android platform, so this app can send and receive signals via a Bluetooth communication protocol. The Nano board then updates the light status accordingly and forward the update status to the cloud with the help of Wi-Fi Shield. Also, for a sudden urgency, the cloud server can also send the signal status update via the same Wi-Fi module and then the Nano board forwards the status to the policeman app.

\subsection{Cloud Automated Suggestions}

The cloud server acts as central distribution module. As all the components of traffic manipulation system are connected to the server via internet media, so it can get all of the status updates from them individually or can send necessary status update to them with the help of the same media. Also, for tracking records, this module maintains database to store data obtained from or data provided to all possible nodes meaning mobile apps as well as embedded IoT lamp posts.

Assigning a lane, for either clearance or halt is a complex operation as it is considered both the number of vehicles is there on the path ways to move about as well as the required minimum time for waiting. Thus, server takes this pressure to generate an optimized solution as a suggestion to the traffic policemen. Also, the server considers an emergency clearance for Ambulance or Fire Bridged or VIP or CIP etc.

Here, 0 index of emergency array holds the flag for any emergency request inserted by the concern person and index 1 to onwards hold the lane number, priority of the emergency etc. The required algorithm to do this job by the server is mentioned below:

var emergency[]

var traffic[]

var density[], time[]

While TRUE

$$
\begin{aligned}
& \text { traffic[] = getTraffic }() \\
& \text { density[] = getDensity (traffic[]) } \\
& \text { time[] = getTime(traffic[] }) \\
& \text { emergency[] = getEmergency() }
\end{aligned}
$$

$$
\begin{aligned}
& \begin{array}{l}
\text { If emergency[0] is TRUE Then } \\
\text { getlane(emergency[]) }
\end{array} \\
& \text { Else } \quad \text { getlane(density[], time[]) } \\
& \text { If End }
\end{aligned}
$$

While End

\subsection{Mobile Applications}

This traffic manipulation system requires two different mobile applications to data input and getting traffic status update via internet media. One of the mobile apps is considered for data input by the traffic policemen and other one is to getting the traffic status update from server.

\subsubsection{TRAFFIC SIGNAL APP}

This application can be accessed by the traffic policemen or the authorized persons who are capable to change the traffic signal status on an assigned IoT traffic lamp post only. As this app has a direct access on the traffic signal status, so it requires authentication credentials. It takes the user command as an input and forward that command to the embedded lamp post via Bluetooth and also forward the same status to the server via Wi-Fi or mobile data as required. Required algorithm is mentioned below:

var nowOpen $=$ NULL

var DEVICE $=$ THIS

While TRUE

nowOpen $=$ getInput $($ DEVICE $)$

updateLampPost(nowOpen)

While End

$$
\text { updateServer(nowOpen) }
$$

\subsubsection{TRAFFIC STATUS APP}

This application can be accessed by any person who is even not passing the road at that moment. It is dedicated to present the road map along with vehicular information including the number of vehicles is passing currently, their average speed. It also provides the number of signals along with their estimated waiting time from the current location to destination.

Depending on the density of the traffic, this app generates an alternative pathway to reach in the destination in the shortest possible time. This application requires device location from the mobile handset itself as an input along with the traffic density, time and number of signals posts on the way. All of these data help to produce the traffic update map. To perform this task, required algorithm is mentioned below:

var myLocation[]

var lampPosts[], traffic[]

var density[], time[]

While TRUE

$$
\begin{aligned}
& \text { myLocation[] = getLocation(DEVICE) } \\
& \text { locationStatus(myLocation) } \\
& \text { lampPosts[] = getLampPosts( ) } \\
& \text { traffic[] = getTraffic() } \\
& \text { density[] = getDensity(traffic[]) } \\
& \text { time[] = getTime(traffic[]) } \\
& \text { getStatus(myLocation[], density[], time[]) }
\end{aligned}
$$


getDirection(myLocation[], lampPosts[])

While End

\section{OPERATION AND CASE STUDY}

Operational activities have been observed on basis of a fourway traffic control system on a single unit. Two of the cases have been explained below on basis of data inserted into the database with real timestamps:

\subsection{TRAFFIC LAMP POST}

The embedded IoT lamp post takes input both from the policemen as well as from the cloud server. Also, the execution of a signal is traced and updated to the cloud server according to their device ID. In a four-way cross point total four lamp posts (LP) is required and they are named as East (E), South (S), West (W) \& North (N). In the following table, four different statuses have been recorded on four LPs at an arbitrary moment in five different times. Also, the colors of an individual lamp post are mentioned as Red (R), Green $(\mathrm{G})$ and yellow (Y).

Table 1: 4-way crossing lamp posts.

\begin{tabular}{|c|c|c|c|c|c|}
\hline Case & LPE & LPS & LPW & LPN & Time Stamp \\
\hline 1 & R & Y & R & G & 1540367553 \\
\hline 2 & Y & G & R & R & 1540367696 \\
\hline 3 & G & R & Y & R & 1540367812 \\
\hline 4 & R & R & G & Y & 1540368012 \\
\hline 5 & R & Y & R & G & 1540368112 \\
\hline
\end{tabular}

\subsection{TRAFFIC POST PROTOTYPE}

In the mentioned four-way road traffic cross point, pathways are of two types named Up (U) and Down (D). For a certain G-Signal, only one up line is open to move forward at a time. Also, all the down lines are open to pass away the cross at the same time. All these operations are observed by the cloud server along the time to analyze the road traffic pressure from road to road.

Table 2: 4-way vehicle movement.

\begin{tabular}{|c|c|c|c|c|c|}
\hline Case & ELU & SLU & WLU & NLU & Time Stamp \\
\hline 1 & $\mathrm{C}$ & $\mathrm{C}$ & $\mathrm{C}$ & $\mathrm{O}$ & 1540367553 \\
\hline 2 & $\mathrm{C}$ & $\mathrm{O}$ & $\mathrm{C}$ & $\mathrm{C}$ & 1540367696 \\
\hline 3 & $\mathrm{O}$ & $\mathrm{C}$ & $\mathrm{C}$ & $\mathrm{C}$ & 1540367812 \\
\hline 4 & $\mathrm{C}$ & $\mathrm{C}$ & $\mathrm{O}$ & $\mathrm{C}$ & 1540368012 \\
\hline 5 & $\mathrm{C}$ & $\mathrm{C}$ & $\mathrm{C}$ & $\mathrm{O}$ & 1540368112 \\
\hline
\end{tabular}

In the table mentioned above, four different statuses have been recorded on basis of total eight path ways in 5 different cases. The required time is varying, due to the consideration of number of vehicles with times.



Fig 6: Time sharing of vehicles in different lanes.

Time sharing of vehicles movement by each of the Up lines is used to present pie-chart mentioned in above. Here the Blue color presents NLU, Red color presents SLU, Green color presents ELU and Violet color presents WLU.

\section{CONCLUSION \& FUTURE WORK}

As the proposed system allows manual input from the traffic police men, so the jam in the road is till depending on them, but smart people can handle a situation smartly. Also, all the modules require continues internet media connection as IoT is introduced here. Failure of internet connection may collapse the entire traffic network. Introducing embedded IoT traffic lamp post also helps to make decision from remotely but till security issue is a big concern here.

This research includes all possible emergency situations that arise in everyday life. The policemen are getting notified the density of vehicles on different roads and traffic cross points, thus they can decide sharply that what needs to be done to avoid traffic jam. And also, general peoples are capable to observe the traffic signal status update with map in their handheld computers and smart watches or glasses.

The number of vehicles is increasing widely in every day. Even a smart road traffic manipulation system is not that smart with this rapid increment of vehicles. Thus, tracking of all kind of vehicles with notification status to move or not to move could be the possible smartest traffic manipulation solution. Then it could be possible to manage road traffic without the intervention of human traffic policemen.

\section{REFERENCES}

[1] I-Vongsingthong, S.; Smanchat, S. (2014). "Internet of Things: A review of applications \& technologies" (PDF). Suranaree Journal of Science and Technology.

[2] Internet: http://www.quizee.club/url/1n

[3] Work station computer: http://www.quizee.club/url/1m

[4] CCTV: http://www.quizee.club/url/11

[5] Embedded System: http://www.quizee.club/url/1h

[6] Elizabeth Basil and Prof. S. D. Sawant, "IoT based Traffic Light Control System using Raspberry Pi, International Conference on Energy", Communication, Data Analytics and Soft Computing (ICECDS-2017).

[7] Luminous: http://www.quizee.club/url/1o

[8] LDR: http://www.quizee.club/url/1j

[9] IR: http://www.quizee.club/url/1k

[10] Solar cells: http://www.quizee.club/url/1i 
[11] Mohd. Saifuzzaman, Nazmun Nessa Moon and Fernaz Narin Nur, "IoT Based Street Lighting and Traffic Management System", 2017 IEEE Region 10 Humanitarian Technology Conference (R10-HTC)

[12] WI-FI transceiver shield: http://www.quizee.club/url/1p

[13] Android OS: https://www.android.com/
[14] iOS: https://www.apple.com/ios/ios-12/

[15] Apache Server: http://httpd.apache.org/

[16] Arduino Nano Board: http://www.quizee.club/url/1g

[17] HC-05 Bluetooth Shield: http://www.quizee.club/url/1f

[18] ESP-8266 Wi-fi Shield: http://www.quizee.club/url/1e 